\title{
Distinct roles of Smad pathways and p38 pathways in cartilage-specific gene expression in synovial fibroblasts
}

\author{
Hiroaki Seto,, ${ }^{1,2}$ Satoshi Kamekura, ${ }^{1}$ Toshiki Miura, ${ }^{1}$ Aiichiro Yamamoto, ${ }^{1}$ Hirotaka Chikuda, ${ }^{1}$ \\ Toru Ogata, ${ }^{1}$ Hisatada Hiraoka, ${ }^{1}$ Hiromi Oda, ${ }^{1}$ Kozo Nakamura, ${ }^{1}$ Hisashi Kurosawa, ${ }^{2}$ Ung-il Chug, ${ }^{3}$ \\ Hiroshi Kawaguchi, ${ }^{1}$ and Sakae Tanaka'
}

1Department of Orthopaedic Surgery, Faculty of Medicine, The University of Tokyo, Tokyo, Japan. 2Department of Orthopaedics, Juntendo University School of Medicine, Tokyo, Japan. ${ }^{3}$ Division of Tissue Engineering, University of Tokyo Hospital, Tokyo, Japan.

\begin{abstract}
The role of TGF- $\beta$ /bone morphogenetic protein signaling in the chondrogenic differentiation of human synovial fibroblasts (SFs) was examined with the adenovirus vector-mediated gene transduction system. Expression of constitutively active activin receptor-like kinase $3\left(\mathrm{ALK} 3{ }^{\mathrm{CA}}\right)$ induced chondrocyte-specific gene expression in SFs cultured in pellets or in SF pellets transplanted into nude mice, in which both the Smad and $\mathrm{p} 38$ pathways are essential. To analyze downstream cascades of ALK3 signaling, we utilized adenovirus vectors carrying either Smad1 to stimulate Smad pathways or constitutively active MKK6 (MKK6 ${ }^{\mathrm{CA}}$ ) to activate $\mathrm{p} 38$ pathways. Smad1 expression had a synergistic effect on ALK3 ${ }^{\mathrm{CA}}$, while activation of $\mathrm{p} 38$ MAP kinase pathways alone by transduction of $\mathrm{MKK}^{\mathrm{CA}}$ accelerated terminal chondrocytic differentiation, leading to type $X$ collagen expression and enhanced mineralization. Overexpression of Smad1 prevented MKK6 ${ }^{\mathrm{CA}}$-induced type $X$ collagen expression and maintained type II collagen expression. In a mouse model of osteoarthritis, activated $\mathrm{p} 38$ expression as well as type $\mathrm{X}$ collagen staining was detected in osteochondrophytes and marginal synovial cells. These results suggest that SFs can be differentiated into chondrocytes via ALK3 activation and that stimulating Smad pathways and controlling $\mathrm{p} 38$ activation at the proper level can be a good therapeutic strategy for maintaining the healthy joint homeostasis and treating degenerative joint disorders.
\end{abstract}

\section{Introduction}

Injury to the articular cartilage occurs under various pathological conditions such as trauma, inflammation, and aging (1), and cartilage injury is followed by osteoarthritic changes of the affected joints. Osteoarthritis is the most common degenerative joint disorder, affecting nearly half of the elderly population. Osteoarthritis is characterized by degradation of articular cartilage and overgrowth of cartilage and bone, known as osteophytes, at the periphery of the articular surface, which results in pain and loss of joint function $(1,2)$. Microscopically, loss of proteoglycan and fibrillation of the articular surface are observed at the early stage of arthritis. At later stages, clefts are formed, and at the end stage, erosive changes in the articular cartilage appear. The high prevalence of this disease results in high costs for treating patients, and therefore the development of good therapeutics for osteoarthritis is a matter of great urgency. Because of the limited capacity of spontaneous healing, the regeneration of intact articular cartilage is one of the most challenging issues in the orthopedic field $(3,4)$. Transplantation of autologous chondrocytes or mesenchymal progenitor cells and autogenous osteochondral transplantation (mosaicplasty) have been successfully utilized for the repair of focal osteochondral defects $(3,5-11)$. However, the application of these

Nonstandard abbreviations used: anterior cruciate ligament (ACL); bone morphogenetic protein (BMP); constitutively active activin receptor-like kinase 3 (ALK3 $\left.{ }^{\mathrm{CA}}\right)$; constitutively active MKK6 (MKK6 $\left.{ }^{\mathrm{CA}}\right)$; hemagglutinin (HA); matrix metalloproteinase (MMP); medial meniscus (MM); osteoarthritis (OA); receptorregulated Smad (R-Smad); synovial fibroblast (SF); TGF- $\beta$-activating kinase 1 (TAK1). Conflict of interest: The authors have declared that no conflict of interest exists. Citation for this article: J. Clin. Invest. 113:718-726 (2004). doi:10.1172/JCI200419899. technologies is limited to small defects due to the difficulty of obtaining a sufficient amount of cells or tissues.

Synovium is a thin tissue lining the nonarticular surfaces of diarthrodial joints (12). Synovial tissues contain various types of cells, including type A cells, macrophage lineage cells, and type B cells, which are specialized synovial fibroblasts (SFs). It is now widely recognized that synovial tissues are involved primarily in the pathogenesis of arthritic joint disorders such as rheumatoid arthritis by producing the matrix-degenerating enzymes cystein proteases and matrix metalloproteinases (MMPs) and the proinflammatory cytokines interleukin-1 (IL- 1 ) and tumor necrosis factor- $\alpha$ $(\mathrm{TNF}-\alpha)(12)$. We previously reported that SFs express a high level of receptor activator of NF- $\mathrm{KB}$ ligand, the osteoclast differentiation factor belonging to the TNF- $\alpha$ superfamily (13). In contrast to such catabolic actions, there is accumulating evidence that synovial cells have anabolic effects, leading to bone and cartilage production. Hunziker and Rosenburg reported that synovial cells can migrate into partial-thickness articular cartilage defects, where they proliferate and subsequently deposit a scar-like tissue (14). Nishimura et al. (15) demonstrated SFs show chondrogenic differentiation after being cultured in the presence of TGF- $\beta$, and de Bari et al. recently demonstrated that multipotent mesenchymal stem cells could be isolated from human synovial tissues, which differentiated into chondrocytes as well as osteoblasts, adipocytes, and myotubes under proper culture conditions $(16,17)$. Another dramatic clinical manifestation of the chondrogenic potential of synovial tissues is synovial chondromatosis, a tumor-like disorder characterized by the formation of multiple cartilaginous nodules, which is believed to be benign reactive metaplasia of synovial cells (18). These observations 
lead us to speculate that synovial tissues contain multipotent cells with chondrogenic potential that might be involved in the repair process of articular cartilage defects and therefore might provide a good source for engineering articular cartilage.

There is accumulating evidence that TGF- $\beta$ superfamily cytokines play an essential role in bone and cartilage development. Wozney and coworkers (19) reported that bone morphogenetic proteins (BMPs) induce early cartilage formation, and various studies have shown that TGF- $\beta$ induces chondrocytic differentiation of undifferentiated mesenchymal cells (20-22). In the present study, we analyzed the role of TGF- $\beta /$ BMP signaling on chondrogenic differentiation of human SFs by using the adenovirus vector-mediated gene transduction system. The introduction of an activated mutant of ALK3 (constitutively active activin receptor-like kinase 3 [ALK3 $\left.{ }^{\mathrm{CA}}\right]$ ), also known as BMP type IA receptor, induced chondrocyte-specific marker expression in the cells. ALK3 signaling involves two different downstream cascades, the Smad pathway and the p38 MAP kinase pathway. We used a combination of adenoviral gene delivery and chemical inhibition to analyze the role of these two signaling cascades in inducing differentiation of SF cells toward a chondrocyte phenotype and found that both pathways are essential for chondrogenic differentiation. Interestingly, activation of p38 pathways alone induced markers of terminal chondrocyte differentiation, type $\mathrm{X}$ collagen expression and mineralization, which was suppressed by Smad 1 coexpression. These results suggest that both the Smad and p38 pathways are necessary for chondrogenic differentiation of SFs and that the balance between these two pathways determines the stage of differentiation.

\section{Methods}

Chemicals and antibodies. Alpha-modified minimum essential medium $(\alpha-M E M)$ was purchased from Gibco BRL, Life Technologies Inc. (Rockville, Maryland, USA), and fetal bovine serum (FBS), from Sigma-Aldrich (St. Louis, Missouri, USA). Anti-p38 MAPK and anti-phospho-p38 MAPK (Thr180/Tyr182) were obtained from Cell Signaling Inc. (Cummings Center, Beverly, Massachusetts, USA). Anti-Flag was purchased from Sigma-Aldrich, and antihemagglutinin (anti-HA) was from Santa Cruz Biotechnology Inc. (Santa Cruz, California, USA). Anti-phospho-Smad1/5/8, which recognizes the phosphorylated form of Smad1, Smad 5, and Smad8, and anti-phospho-Smad2 were from Cell Signaling Inc. Anti-type II collagen was purchased from Oncogen (Boston, Massachusetts, USA) and anti-type X collagen was from LSL Co. (Cosmo Bio, Tokyo, Japan). Other chemicals and reagents used in this study were of analytical grade.

Isolation of SFs from buman synovial tissues. Synovial cells were obtained as previously described $(13,23,24)$. In brief, with enzymatic digestion, human synovial cells were isolated from synovial tissues of the knee joints of ten rheumatoid arthritis patients (37-75 years of age; mean, 60.3 years of age) at the time of total knee arthroplasty operations. Written informed consent for subsequent experiments was obtained from each patient. Cells were suspended in $\alpha$-MEM containing $10 \%$ FBS and were cultured in monolayers. After three to five passages, subcultured cells were composed of morphologically uniform fibroblastic cells (SFs) that were free of macrophages. They were infected with adenovirus vectors and cultured in pellets ("pellet culture"). Primary chondrocytes were obtained from articular cartilage resected during the surgeries. Cartilage was minced finely in phosphate-buffered saline (PBS), and chondrocytes were isolated by sequential digestion at $37^{\circ} \mathrm{C}$ with
$0.25 \%$ (weight/volume) trypsin for 30 minutes and with $2 \mathrm{mg} / \mathrm{ml}$ of clostridial collagenase in $\alpha$-MEM containing $10 \%$ FBS and antibiotics (penicillin at $100 \mu \mathrm{g} / \mathrm{ml}$ and streptomycin at $100 \mu \mathrm{g} / \mathrm{ml}$ ) overnight on an orbital shaker. Cells were isolated by centrifugation and were resuspended in $\alpha$-MEM with $10 \%$ FBS. Cells were cultured in monolayers for 1 day and then subjected to RNA isolation.

Constructs and gene transduction. The recombinant adenovirus vectors carrying various molecules that modulate TGF- $\beta$ superfamily signaling pathways, that is, HA-tagged constitutively active TGF- $\beta /$ BMP type I receptors (ALK3 ${ }^{\mathrm{CA}}, \mathrm{ALK} 5^{\mathrm{CA}}$, and $\mathrm{ALK} 6^{\mathrm{CA}}$ ), constitutively active MKK6 (MKK6 ${ }^{\mathrm{CA}}$ ), Flag-tagged Smad1 and Smad6 with CAG [cytomegalovirus IE enhancer + chicken $\beta$-actin promoter + rabbit $\beta$-globin poly(A) signal] promoter, were generated by the DNA-terminal protein complex method (25-27). SFs were infected with adenovirus vectors following a method previously described (13). In short, subconfluent SFs were incubated with a small amount of medium $(\alpha$-MEM without serum) that contained the recombinant adenoviruses for 2 hours at $37^{\circ} \mathrm{C}$ at the indicated multiplicity of infection (MOI) and then with 10 times more medium to which $10 \%$ FBS had been added. Infected cells were cultured for additional 3 days for assessment of chondrogenic gene expression or were subjected to pellet culture 24 hours after the infection for histological examination.

Pellet cultures of isolated SFs. After 24 hours of viral infection, adherent cells were trypsinized and cells numbers were ascertained. Aliquots of $5 \times 10^{5}$ cells were spun down at $500 \mathrm{~g}$ in 15 -ml polypropylene conical tubes in $5 \mathrm{ml}$ of $\alpha$-MEM with ascorbate 2 -phosphate $(0.1 \mathrm{mM})$ and $10 \% \mathrm{FBS}$. The cells were incubated at $37^{\circ} \mathrm{C}$ in $5 \% \mathrm{CO}_{2}$. Within 24 hours after incubation, the cells formed a single, freefloating pellet. The medium was changed every $2-3$ days, and duplicate pellets were harvested after 3 and 7 days for real-time-PCR and Northern blotting and after 3 and 5 weeks for histological and immunohistochemical analysis. For visualization of the chondrogenic differentiation in vivo pellets were transplanted subcutaneously into $n u / n u$ BALB mice (nude mice) after 3 days of pellet culture. Mice were sacrificed 5 weeks after transplantation and the pellets were recovered and subjected to toluidine blue staining as well as immunostaining with anti-type II collagen.

Immunoblotting. All the extraction procedures were performed at $4^{\circ} \mathrm{C}$ or on ice. Cells were washed with PBS and then lysed by the addition of TNE buffer (1\% NP-40, 10 mM Tris-HCl, pH 7.8, 150 $\mathrm{mM} \mathrm{NaCl}, 1 \mathrm{mM}$ EDTA, $2 \mathrm{mM} \mathrm{Na}_{3} \mathrm{VO}_{4}, 10 \mathrm{mM} \mathrm{NaF}$, and $10 \mu \mathrm{g} / \mathrm{ml}$ aprotinin). Lysates were prepared by centrifugation at $10,000 \mathrm{~g}$ for 20 minutes. An equal amount $(15 \mu \mathrm{g})$ of proteins was separated by electrophoresis on $10 \%$ SDS-polyacrylamide gels. After electrophoresis, proteins were electronically transferred onto a nitrocellulose membrane. Immunoblotting with specific antibodies was performed with ECL Western blotting reagents (Amersham Co., Arlington Heights, Illinois, USA) according to the conditions recommended by the supplier.

Histology and immunostaining. Pellet cultures were fixed with $3.7 \%$ formaldehyde, embedded in paraffin, and cut into sections $4 \mu \mathrm{m}$ in thickness. Representative sections were subjected to Alcian blue staining, Alizarin red staining, and immunohistochemistry. Alcian blue staining was performed according to the protocol described previously (28). Briefly, after deparaffinization, sections were stained with $0.5 \%$ Alcian blue 8GX (Wako, Osaka, Japan) in $0.1 \mathrm{~N} \mathrm{HCl}$ for 1 hour. Mineralization was assessed by Alizarin red staining. In brief, sections were immersed in Alizarin red solution ( $40 \mathrm{mM}$, at $\mathrm{pH} 4.0$ ) for $8 \mathrm{~min}$ utes at room temperature, and nonspecific staining was removed by several washes in distilled water. For immunostaining with anti-type 
II collagen or anti-type X collagen, we utilized a CSA Kit (DAKO, Carpinteria, California, USA) following the manufacturer's protocol.

Total RNA extraction and real-time PCR. Total RNA was isolated from SFs with ISOGEN (Wako) following the supplier's protocol. Complementary DNA (cDNA) was synthesized from $1 \mu \mathrm{g}$ of total RNA with the Superscript II reverse transcriptase kit (Invitrogen, Carlsbad, California, USA). For real-time PCR, the ABI Prism Sequence Detection System 7000 was used. Primers were designed based on sequences obtained from GenBank and amplicons of 50-250 base pairs with a melting temperature of between $55^{\circ} \mathrm{C}$ and $60^{\circ} \mathrm{C}$ were selected. Aliquots of first-strand cDNA $(1 \mu \mathrm{g})$ were amplified with the QuantiTect SYBER Green PCR Kit (Qiagen, Valencia, California, USA) under the following conditions: initial denaturation for 10 minutes at $94^{\circ} \mathrm{C}$ followed by 40 cycles consisting of 15 seconds at $94^{\circ} \mathrm{C}$ and 1 minute at $60^{\circ} \mathrm{C}$. Data analysis consisted of fold induction, and the expression ratio was calculated from the differences in threshold cycles at which an increase in reporter fluorescence above a baseline signal could first be detected among three samples and was averaged for duplicate experiments. The primers we utilized in real-time PCR to detect sox9, type II collagen, type X collagen, osteocalcin, osteopontin, and GAPDH were as follows: sox9, 5'-AGAAGGACCACCCGGATTAC-3' and 5'-AAGTCGATAGGGGGCTGTCT3'; type II collagen, 5'-GGTGGCTTCCATTTCAGCTA-3' and 5'-TACCGGTATGTTTCGTGCAG-3'; type X collagen, $5^{\prime}$-AGGAATGCCTGTGTCTGCTT- $3^{\prime}$ and $5^{\prime}$-ACAGGCCTACCCAAACATGA-3'; osteocalcin, 5'-GTGCAGAGTCCAGCAAAGGT-3' and 5'-CGATAGGCCTCCTGAAAGC-3'; osteopontin, 5'-ACAGCCAGGACTCCATTGAC- $3^{\prime}$ and 5'-ACACTATCACCTCGGCCATC- $3^{\prime}$; and GAPDH, 5'-GAAGGTGAAGGTCGGAGTCA-3' and 5' -GAAGATGGTGATGGGATTTC-3'.

Northern blotting. Equal amounts $(15 \mu \mathrm{g})$ of RNA were denatured in formaldehyde, separated by $1 \%$ agarose gel electrophoresis and transferred to a nitrocellulose membrane $\left(\right.$ Hybond $\mathrm{N}^{+}$) (Amersham Pharmacia, Piscataway, New Jersey, USA), followed by ultraviolet crosslinking. ULTRAHyb hybridization solution (Ambion, Austin, Texas, USA) was used according to the manufacturer's protocol. The blots were hybridized with a cDNA probe labeled with $\left[\alpha-{ }^{32} \mathrm{P}\right] \mathrm{dCTP}$ using Ready-To-Go DNA Labeling Beads (Amersham Pharmacia). Rabbit type II collagen and aggrecan probes were generously provided by Yoshiyasu Iwamoto (Thomas Jefferson University, Philadelphia, Pennsylvania, USA). Membranes were washed in $2 \times$ SSC for 15 minutes at $42^{\circ} \mathrm{C}$ and then in $0.1 \times \mathrm{SSC}$ for 30 minutes at $65^{\circ} \mathrm{C}$. For visualization, $\mathrm{X}$-ray film was exposed to membranes overnight at $-80^{\circ} \mathrm{C}$.

Osteoarthritis model mice. Osteoarthritic changes were developed in the knee joint by transection of the anterior cruciate ligament (ACL) and medial meniscus (MM) in C57BL/ 6 mice (mean age, 8 weeks) (29, 30). Briefly, after mice were anesthetized with ketamine and xylazine, a medial parapatellar skin incision was made. The subcutaneous tissues were incised and retracted, along with the articular capsule. The medial compartment of the knee joint was visualized and the ACL and MM were transected with a scalpel, and thereafter the capsule, medial retinaculum, and skin were sutured. Mice were housed in regular individual cages and allowed to exercise. Eight weeks after the surgery, the mice were sacrificed and paraffin-embedded sections of the affected joints were immunostained with anti-type $\mathrm{X}$ collagen and anti-phospho-p38 (Cell Signaling Technology Inc).

\section{Results}

Adenovirus-mediated gene transduction modulates the Smad and $p 38$ pathways in SFs. We previously reported that adenovirus vectors can effi- ciently transduce foreign genes into synovial cells both in vitro and in vivo and that adenovirus infection itself does not affect the phenotypes of the cells (13). We constructed adenovirus vectors to analyze the role of ALK signaling as well as the Smad pathways and p38 pathways, which lie downstream of ALK signaling. SFs were infected with adenovirus vectors carrying various signaling molecules that modulate TGF- $\beta$ superfamily signaling pathways, that is, HA-tagged constitutively active ALK3, ALK5, and ALK6 constitutively active MKK6, and Flag-tagged Smad1 and Smad6, as well as a control virus carrying the $\beta$-galactosidase gene (Lac $Z$ virus), and gene expression was determined by immunoblotting with specific antibodies. As shown in Figure 1, clear induction of the genes encoding ALK3 ${ }^{\mathrm{CA}}$, ALK5 ${ }^{\mathrm{CA}}$ and ALK6 ${ }^{\mathrm{CA}}$ was observed by immunoblotting with anti-HA (Figure 1A), and Smad1 and 6, by anti-Flag (Figure 1B). ALK3 ${ }^{\mathrm{CA}}$ or ALK $6^{\mathrm{CA}}$ overexpression induced phosphorylation of Smad1, Smad5, and Smad8 in SFs, and ALK5 ${ }^{\mathrm{CA}}$-transduced cells showed Smad2 phosphorylation (Figure $1 \mathrm{~A}$ ). MKK6 ${ }^{\mathrm{CA}}$ virus infection specifically activated $\mathrm{p} 38$ pathways in SFs, and the pathways were also activated in ALK3 $3^{\mathrm{CA}}$-transduced cells as determined by Western blotting with anti-phospho-p38 (Figure 1C). The increased p38 phosphorylation induced by either $\mathrm{ALK} 3^{\mathrm{CA}}$ or $\mathrm{MKK} 6^{\mathrm{CA}}$ overexpression was suppressed by the p38-selective inhibitor SB203580.

Induction of chondrocyte-specific gene expression by ALK3 ${ }^{C A}$ transduction in pellet cultures of SFs. To determine the effects of these transduced gene products on chondrocyte-specific gene expression in SFs, we subjected infected cells to pellet culture. After 7 days of culture, clear induction of type II collagen and aggrecan genes was observed in $\mathrm{ALK} 3^{\mathrm{CA}}$-transduced cultures by both Northern blot analysis (Figure 2A) and real-time PCR (Figure 2, B and C). Expression of these genes was also observed in ALK6 ${ }^{\mathrm{CA}}$-transduced cultures, albeit less efficiently, as shown in Figure 2, B and C, by real-time PCR. Contrary to the strong chondrogenic effects of ALK3 ${ }^{\mathrm{CA}}$ virus, expression

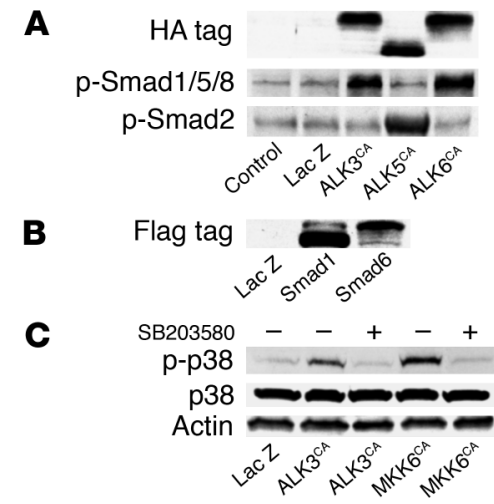

\section{Figure 1}

Modulation of intracellular signaling pathways by adenovirus vector-mediated gene transduction into SFs. (A) SFs at passage 3 were transduced with HA-tagged constitutively active ALK3, ALK5, and ALK6, and the expressed products were detected by immunoblotting after 2 days of viral infection. Expression of these genes was detected by immunoblotting with anti-HA and phospho-Smad1, -Smad 5, and Smad8 ( $p$-Smad1/5/8) was observed in cells expressing ALK3 ${ }^{C A}$ or ALK6 ${ }^{C A}$, and p-Smad2, in cells expressing ALK5 ${ }^{\mathrm{CA}}$. (B) Expression of Smad1 and 6 in SFs was determined by anti-Flag. (C) Adenovirus vector-mediated ALKCA or MKK6 ${ }^{\text {CA }}$ expression specifically activated p38 pathways in SFs, as determined by Western blotting with anti-phospho-p38 (p-p38). The increased p38 phosphorylation induced by ALK3 ${ }^{C A}$ or MKK6 CA overexpression was suppressed by the p38-selective inhibitor SB203580. 

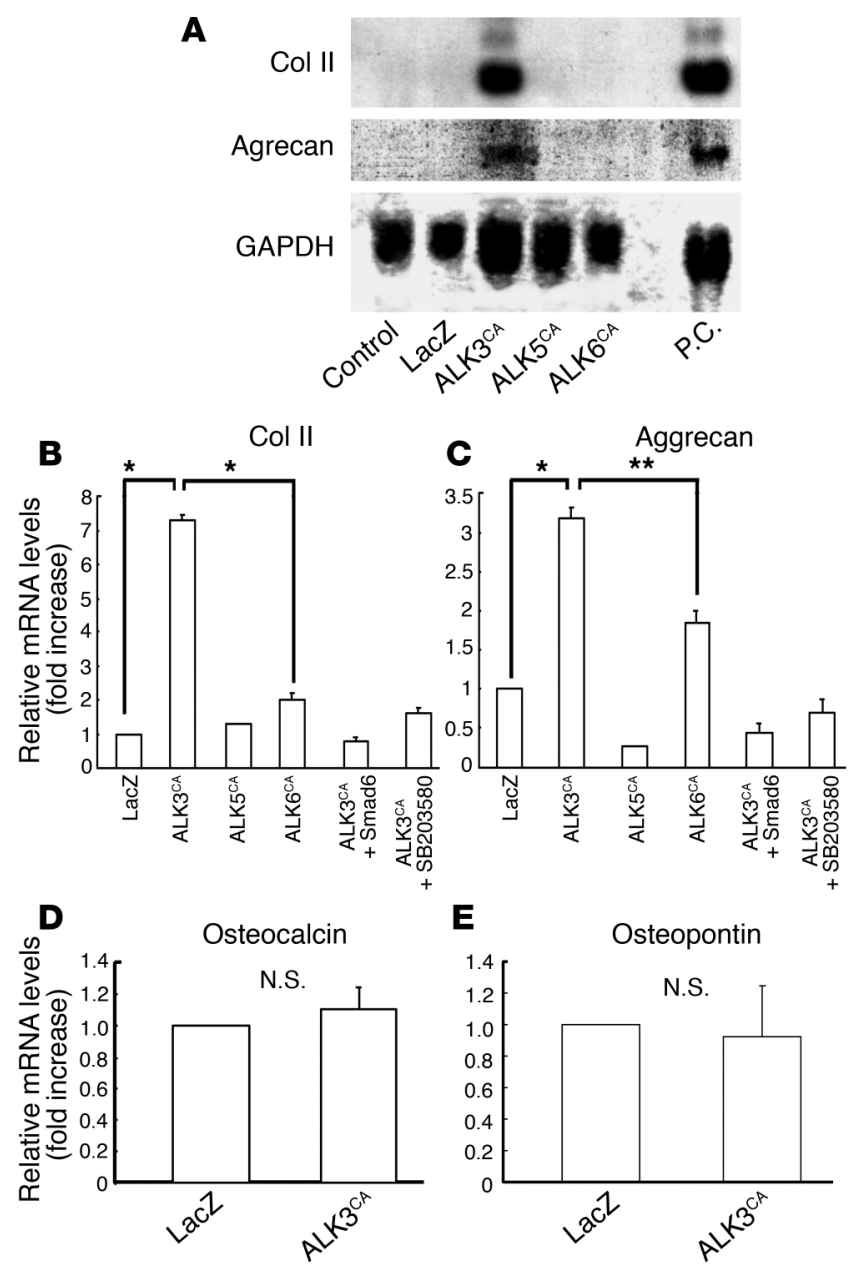

of osteocalcin or osteopontin was hardly detectable in the cells (Figure 2, C and D), indicating that hypertrophic and osteogenic differentiation were somehow blocked in these cultures. In contrast, neither type II collagen nor aggrecan gene expression was observed in ALK5 ${ }^{\mathrm{CA}}$ virus-infected cells (Figure 2, A-C). Type II collagen and aggrecan expression induced by ALK $3^{\mathrm{CA}}$ transduction was completely suppressed by coexpression with Smad6 or by SB203580 (Figure 2, B and C).

ALK3 gene transduction increases Alcian blue-positive matrix and type II collagen deposition in pellet cultures of SFs. For histological analysis, cells were subjected to pellet culture 24 hours after the viral infection. After 3 weeks of pellet culture, cells were fixed and examined by Alcian blue staining (Figure 3, A, D, G, and I) and Alizarin red staining and type II collagen immunostaining (Figure 3, B, E, G, and J) and type $\mathrm{X}$ collagen immunostaining (Figure 3, C and F). ALK3 3 CA virus-infected cultures showed cartilageous matrix production that was strongly positive for Alcian blue staining (Figure 3D), while no positive staining was observed in LacZ virus-infected cultures (Figure $3 \mathrm{~A}$ ) or ALK5 ${ }^{\mathrm{CA}}$ virus-infected cultures (Figure $3 \mathrm{G}$ ), and only weak staining was observed in ALK6 ${ }^{\mathrm{CA}}$ virus-infected cultures (Figure $3 \mathrm{H})$. No Alizarin red staining was observed in ALK3 ${ }^{\mathrm{CA}}$-infected cultures (not shown), indicating that mineralization associated with osteogenic differentiation was not induced. ALK3 $3^{\mathrm{CA}}$ virus-infected SFs showed an oval shape, morphologically reminiscent of chondrocytes (Figure 3D). Immunostaining with

\section{Figure 2}

Effects of ALK3 ${ }^{\mathrm{CA}}$, ALK5 ${ }^{\mathrm{CA}}$, and ALK6 ${ }^{\mathrm{CA}}$ expression on chondrocyte-specific gene expression in SFs. (A-E) Gene expression in SFs, as determined by Northern blot analysis (A) and real-time PCR analysis (B-E). Subconfluent monolayer SF cultures were infected with adenovirus vectors and they were then subjected to pellet culture 24 hours after viral infection; mRNA extracted from the pellets after 7 days of culture was then analyzed. Expression of type II collagen (Col II) and aggrecan was clearly induced in ALK3 ${ }^{\mathrm{CA}}$-expressing cultures, as shown by Northern blot analysis (A) and real-time PCR analysis (B and $\mathbf{C})$; this was suppressed by Smad6 coexpression and SB203580 (B and C). Expression of type II collagen and aggrecan was also observed in ALK6 CA-expressing cultures, albeit less efficiently, as shown in $\mathbf{B}$ and $\mathbf{C}$ by real-time PCR. Neither the osteocalcin nor the osteopontin gene was induced by ALK3CA virus infection (D and E). P.C., positive control, which represents the Northern blotting using mRNA of primary chondrocytes. N.S., not significant; ${ }^{*} P<0.001 ;{ }^{* \star} P<0.005$ (significantly different).

anti-type II collagen showed positive staining in ALK3 ${ }^{\mathrm{CA}}$ virus-infected pellet cultures (Figure $3 \mathrm{E}$ ) and weak staining in ALK6 ${ }^{\mathrm{CA}}$ virus-infected cultures (Figure $3 \mathrm{H}$ ), while we failed to detect type $\mathrm{X}$ collagen in ALK3 ${ }^{\mathrm{CA}}$ virus-infected cultures (Figure $3 \mathrm{~F}$ ), which suggests an absence of terminal differentiation to hypertrophic chondrocytes. No positive type II collagen immunostaining was detected in LacZ virus-infected cultures (Figure 3B) or ALK5 CA virus-infected cultures (Figure $3 \mathrm{H}$ ).

ALK3 $3^{C A}$-transduced SFs after pellet culture form cartilage matrix in vivo. To study chondrogenic differentiation of SFs in vivo, we subcutaneously transplanted the pellets into nude mice. Mice were sacrificed 3 weeks after the transplantation and the pellets were recovered and subjected to histological analysis. The transplanted SF pellets expressing ALK3 ${ }^{\mathrm{CA}}$ were positively stained for toluidine blue (Figure 4C), which detects proteoglycan components, as does Alcian blue staining. Type II collagen immunostaining was also positive (Figure 4D), indicating the cartilaginous differentiation of the cultures in vivo, while Alizarin red staining was almost undetectable (data not shown). ALK6 ${ }^{\mathrm{CA}}$ expression also induced chondrogenesis, albeit much less prominently (not shown), while neither LacZ (Figure 4, A and B) or ALK5 ${ }^{\mathrm{CA}}$ (not shown) expression could induce chondrogenic phenotypes in the cultures. The histological observation was further confirmed by real-time PCR; expression of type II collagen and aggrecan was significantly higher in ALK3 ${ }^{\mathrm{CA}^{-} \text {-trans- }}$ duced pellets (Figure 4, E and F). These results suggest that ALK3 ${ }^{\mathrm{CA}}$ 

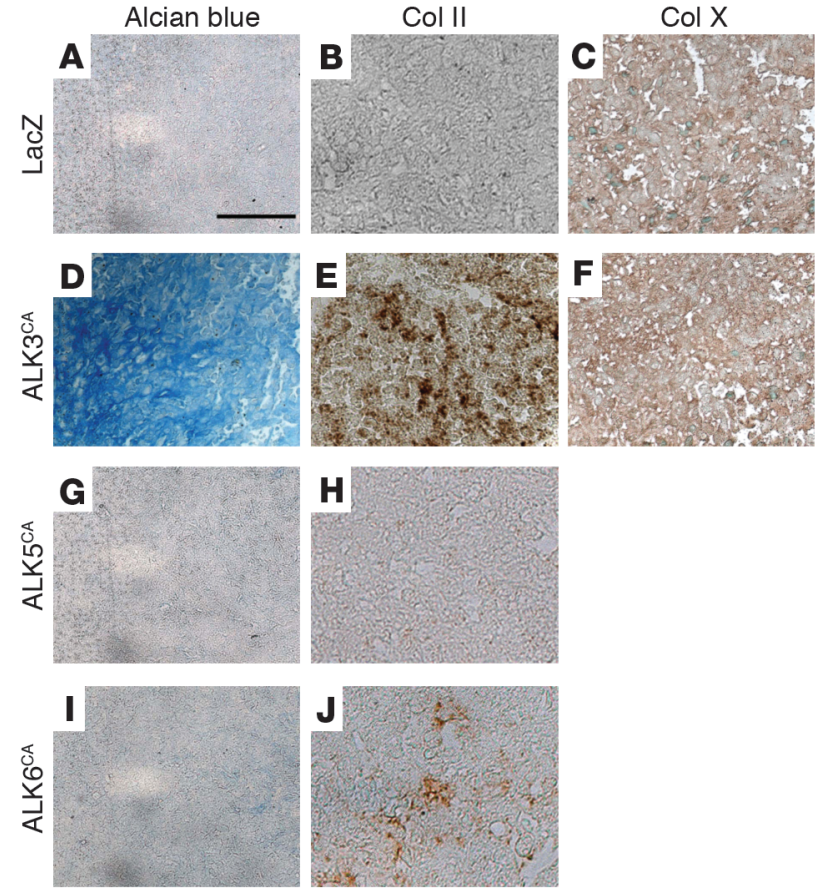

\section{Figure 3}

ALK3 ${ }^{\mathrm{CA}}$ gene transduction increases Alcian blue-positive matrix and type II collagen deposition in pellet cultures of SFs. (A-J) Adenovirus-infected SF pellets were fixed with $3.7 \%$ formaldehyde after 3 weeks of culture and then were subjected to Alcian blue staining (A, D, G, and I) or immunostaining with anti-type II collagen $(\mathbf{B}, \mathbf{E}, \mathbf{H}$, and $\mathbf{J})$ or anti-type $\mathbf{X}$ collagen (Col X) (C and F). Distinct Alcian blue (D) and type Il collagen (E) staining was observed in ALK3 ${ }^{C A}$-expressing cultures. ALK6 ${ }^{C A}$-expressing cultures showed weaker staining ( $\mathbf{I}$ and $\mathbf{J}$ ), and no positive staining was observed in ALK5CA virus-infected ( $\mathbf{G}$ and $\mathbf{H}$ ) or LacZ virus-infected (A and $\mathbf{B}$ ) cultures. No type $\mathbf{X}$ collagen immunostaining was observed in cultures expressing LacZ or ALK3 ${ }^{\mathrm{CA}}$ (C and F). Scale bar: $100 \mu \mathrm{m}$. overexpression was able to target cartilage formation without subsequent bone formation in vivo.

Segregation of ALK signaling pathways. ALK signaling is known to be mediated by both the Smad pathways and MAP kinase pathways, especially the p38 pathways (31-33). We therefore attempted to distinguish the roles of the Smad pathways and p38 pathways from each other using a specific p38 inhibitor or adenovirus vectors. Smad 6 coexpression or treatment of the cultures with the p38 inhibitor SB203580 completely abrogated the chondrogenic gene expression induced by ALK3 $^{\mathrm{CA}}$ (Figure 2, B and C). These results indicate that both the Smad pathways and the p38 MAP kinase pathways are required for the differentiation. Although Smad1 expression alone $(\mathrm{MOI}=20)$ or a small amount of ALK ${ }^{\mathrm{CA}}$ virus $(\mathrm{MOI}=2)$ failed to induce type II collagen expression in SFs, both had synergistic effects, and robust upregulation of type II collagen gene was observed by coinfection of Smad1 virus $(\mathrm{MOI}=20)$ and $\mathrm{ALK} 3^{\mathrm{CA}}$ virus $(\mathrm{MOI}=2)$ (Figure 5A). Interestingly, activation of p38 pathways alone by MKK6 ${ }^{\mathrm{CA}}$ expression in SFs induced rapid induc-

\section{Figure 4}

ALK3 ${ }^{C A}$-transduced SFs form cartilage matrix in vivo. (A-D) Three weeks after transplantation into nude mice, pellets were recovered and stained with toluidine blue (A and $\mathbf{C})$ and immunostained with anti-type II collagen (B and $\mathbf{D})$. Type II collagen immunohistochemistry was shown in the enlarged features of the rectangular area in the toluidine blue staining. Distinct positive staining was observed in ALK3 ${ }^{C A}$-expressing cultures (B and $\mathbf{D})$ in contrast to LacZ virus-infected cultures (A and C). Scale bars: $100 \mu \mathrm{m}$. (E and F) Real-time PCR analysis of type II collagen and aggrecan. Their expression was significantly higher in ALK3 ${ }^{C A}$-expressing pellets than in LacZexpressing pellets. ${ }^{\star} P<0.001$ (significantly different). tion of Sox 9 and type II collagen, which declined rapidly, however, and type $\mathrm{X}$ collagen expression was subsequently increased (Figure 5B). Coexpression of Smad1 together with MKK6 ${ }^{\mathrm{CA}}$ not only reduced type $X$ collagen expression but also maintained type II collagen expression in the cells (Figure 5B). Pellet cultures infected
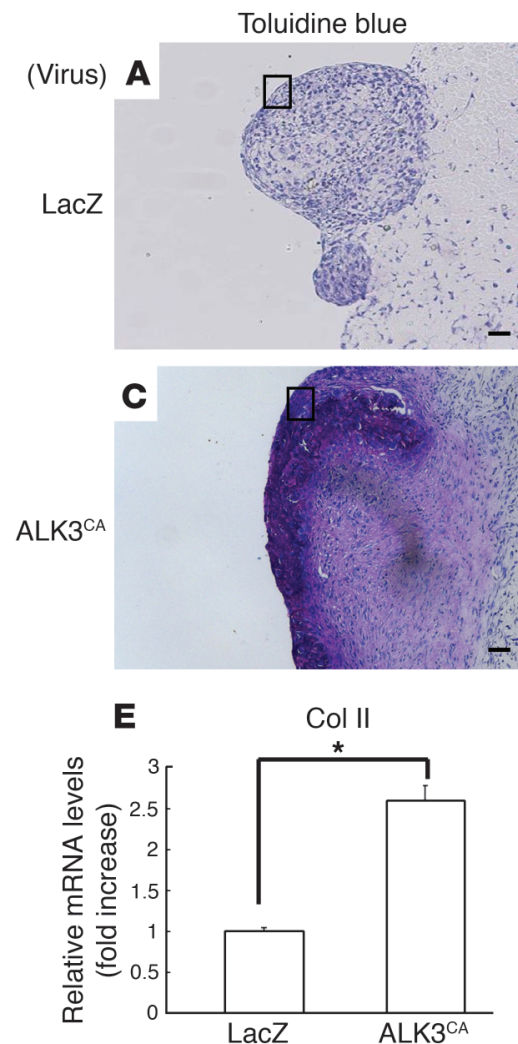
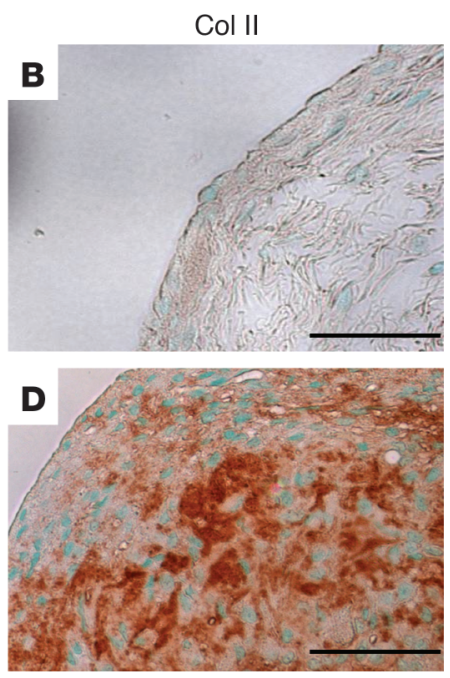

$\mathbf{F}$

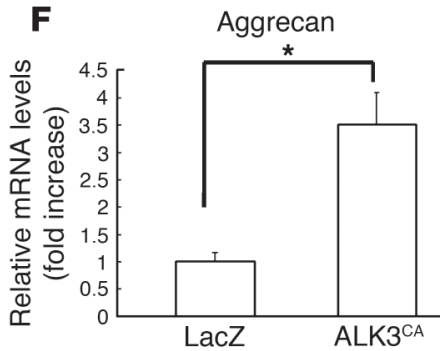


A

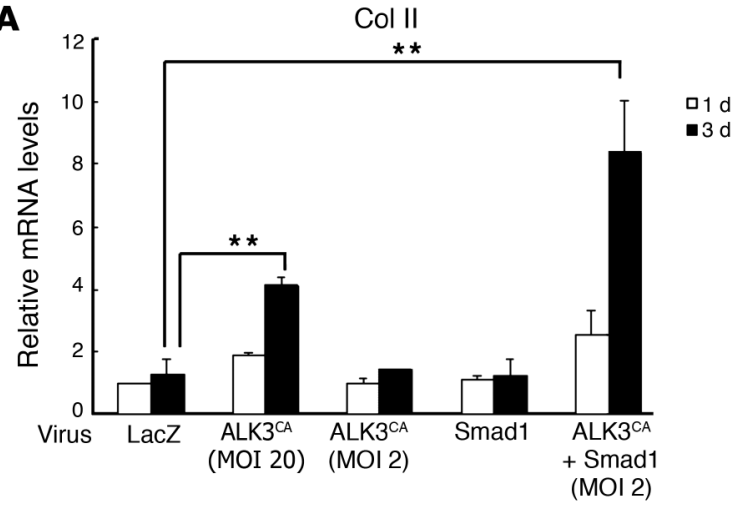

B
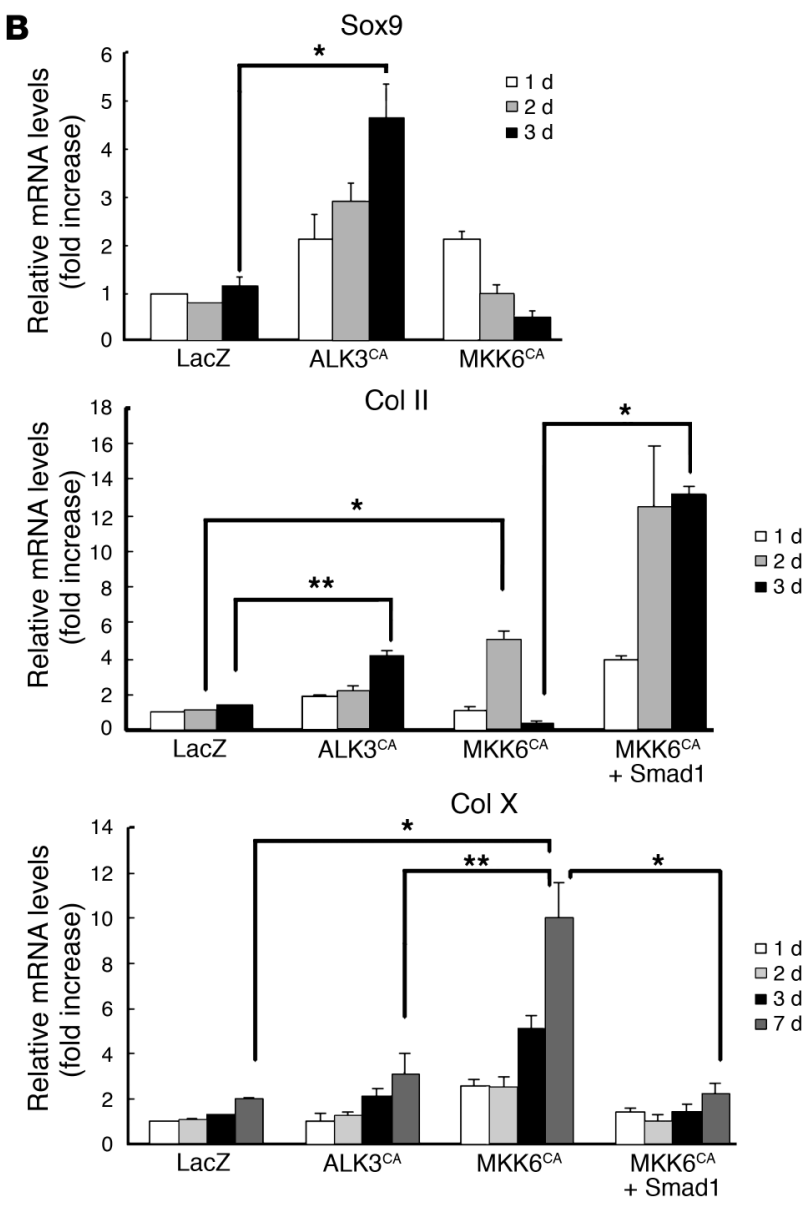

with MKK6 ${ }^{\mathrm{CA}}$ virus were positively stained by type $\mathrm{X}$ collagen immunostaining as well as Alizarin red staining, which was suppressed by Smad1 virus coinfection (Figure 6).

Type $X$ collagen expression and $p 38$ activation in synovial cells in osteoarthritic joints. To examine the role of p38 activation in the development of degenerative changes in the articular cartilage, we next analyzed synovial tissues in the mouse model of osteoarthritis. After ACL and MM resection, the animals developed degenerative joint changes mimicking osteoarthritis. Osteochondrophytes were formed at the posterior edge of the femoral condyle and they were positively stained by anti-type $\mathrm{X}$ collagen as well as toluidine blue (rectangular areas in Figure 7, A and C) 4 weeks after the operation (corresponding to the stage of moderate osteoarthritis).

\section{Figure 5}

Segregation of downstream signaling pathways of ALK3. (A) Synergistic effect of Smad1 expression on the chondrogenic effects of the ALK3CA virus. Expression of Smad1 $(\mathrm{MOI}=20)$ together with ALK3CA virus $(\mathrm{MOI}=2)$ strongly induced expression of type II collagen in SFs. White bars indicate type II collagen expression on day 1 of cultures, and black bars indicate that on day $3 .{ }^{*} P<0.005$ (significantly different). (B) MKK6-p38 pathways promote terminal chondrocytic differentiation of SFs. Mandatory activation of p38 pathways by expression of MKK6CA using adenovirus vectors rapidly activated expression of the Sox9 and type II collagen genes, which rapidly declined, while expression of a terminal chondrocytic differentiation marker, type $X$ collagen, was gradually increased. Adenovirus vector-mediated overexpression of Smad1 together with MKK6 ${ }^{\mathrm{CA}}$ suppressed type $\mathrm{X}$ collagen expression and maintained type II collagen expression in SFs. ${ }^{*} P<0.001$; ${ }^{* *} P<0.005$ (significantly different).

Clusters of migrating synovial cells were observed adjacent to the osteochondrophytes (Figure 7B, arrowheads), where future osteochondrophytes will develop, and they were weakly stained by toluidine blue and anti-type $\mathrm{X}$ collagen at the marginal area between synovium and osteophytes (rectangular areas in Figure 7, B and D). This region was also positively stained by anti-phospho-p38 (Figure 7F). No positive staining was observed in the normal synovium, however (data not shown).

\section{Discussion}

The signaling events leading to chondrogenesis still remain elusive, although there is accumulating evidence that TGF- $\beta$ superfamily cytokines may play an important role (19-22). The receptors of TGF- $\beta$ family members are composed of two different types of serine/threonine kinase receptors, known as type I and type II $(31,34$, 35). Type II receptors are constitutively active kinases and phosphorylate type I receptors, also called ALKs. Type I receptors in turn mediate specific intracellular signaling pathways and therefore determine the specificity of the downstream signaling. So far, seven type I receptors have been identified, ALKs 1-7. ALK3 (BMPR-IA) and ALK6 (BMPR-IB) are structurally similar to each other and function as BMP receptors, while ALK5 and ALK4 work as type I TGF- $\beta$ receptors. Using the adenovirus vector system, Fujii et al. reported that $\mathrm{ALK} 1^{\mathrm{CA}}, \mathrm{ALK} 2^{\mathrm{CA}}, \mathrm{ALK} 3^{\mathrm{CA}}$, and $\mathrm{ALK} 6^{\mathrm{CA}}$ induced osteoblastic differentiation of $\mathrm{C} 2 \mathrm{C} 12$ myoblasts and that $\mathrm{ALK} 3^{\mathrm{CA}}$ or $\mathrm{ALK6} \mathrm{CA}^{\mathrm{CA}}$ introduction induced chondrocytic differentiation of ATDC teratocarcinoma cells (27).

In the present study, we focused on the regulation of chondrogenic differentiation of primary SFs obtained from rheumatoid arthritis patients. SFs have chondrogenic potential $(15,16)$ and can migrate into articular cartilage defects, where they deposit a scar-like tissue as Hunziker et al. pointed out (14), suggesting that SFs have anabolic effects on joint homeostasis and are involved in the restoration process of articular cartilage. We demonstrated that adenovirus vector-mediated ALK3 ${ }^{\mathrm{CA}}$ gene expression induced robust induction of chondrocyte-specific gene expression in SFs in a ligand-independent manner. Clear induction of Sox 9 , a key transcription factor regulating chondrogenesis $(36,37)$, followed by type II collagen and aggrecan expression, was observed in the ALK3 ${ }^{\mathrm{CA}}$-expressing cultures, while type $\mathrm{X}$ collagen was only weakly induced in the cultures and no osteocalcin expression could be found (Figures 2 and 5). Induction of these chondrocyte-specific genes through ALK3 ${ }^{\mathrm{CA}}$ expression was not observed in skin fibroblasts, suggesting the cell specificity of the events (data not 

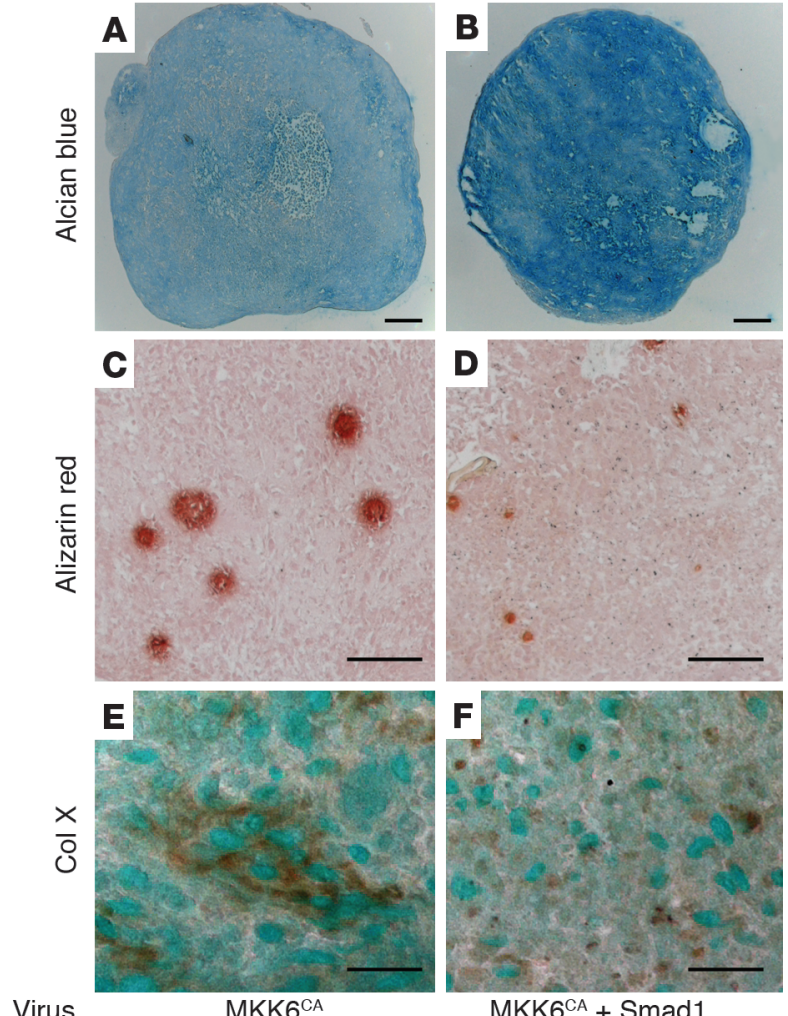

$\mathrm{MKK6}^{\mathrm{CA}}+\mathrm{Smad} 1$ shown). The chondrogenic effect of ALK3 $3^{\mathrm{CA}}$ virus was further confirmed histologically by pellet cultures performed in vitro and in vivo (Figures 3 and 4). Induction of neither the osteoblast markers osteopontin and osteocalcin nor the terminal chondrocyte differentiation markers type $\mathrm{X}$ collagen and mineralization was observed in ALK3 ${ }^{\mathrm{CA}}$-expressing cells (Figures 2, 3, 5, and 6). These results suggest that ALK3 signaling, that is, BMP signaling, has both stimulatory and regulatory roles in chondrogenesis: to induce the chondrogenic differentiation of SFs and at the same time to block their osteoblastic or hypertrophic differentiation. Despite the structural similarity between ALK3 and ALK6, the ALK6 ${ }^{\mathrm{CA}}$ virus was much less efficient in chondrogenesis, the reason for which remains to be clarified. Although many studies have demonstrated a prochondrogenic effect for TGF- $\beta(15,16,20-22)$, we failed to find an anabolic effect for ALK5 ${ }^{\mathrm{CA}}$ which is expected to mimic TGF- $\beta$ signaling, on the chondrogenic differentiation of SFs. We cannot fully explain the discrepancy between our results and those of previous studies, but Robbins and coworkers recently reported that adenovirus vector-mediated TGF- $\beta$ gene transduction into arthritic joints in fact exacerbated cartilage degradation (38), raising the possibility that sustained activation of TGF- $\beta$ signaling, via ALK5, has instead a negative effect on chondrogenesis. Further study will be required to elucidate the difference between TGF- $\beta$ and BMP signaling.

The signaling of TGF- $\beta /$ BMPs is transduced by Smad family members $(31,34,35)$. Receptor-regulated Smads (R-Smads) are direct substrates of type I receptors and are phosphorylated at the C-terminal SSV/MS motif. R-Smads then form heteromeric complexes with common-mediator Smads and translocate into the nuclei, where they regulate transcription of target genes. In addition to Smad pathways, there is evidence that MAP kinase

\section{Figure 6}

Induction of Alizarin red staining and type $X$ collagen in MKK6-transduced SFs in pellet cultures. (A-F) SFs infected with MKK6 ${ }^{\text {CA }}$ virus alone (A, C, and $\mathbf{E}$ ) or together with Smad1 virus (B, D, and F) were subjected to pellet culture. Cultures were fixed with $3.7 \%$ formaldehyde 3 weeks later, and then stained with Alcian blue (A and $\mathbf{B}$ ), Alizarin red (C and $\mathbf{D})$ or anti-type $X$ collagen ( $E$ and $\mathbf{F}$ ). Note the increased Alcian blue staining and the reduced Alizarin red activity and type $X$ collagen immunoactivity with Smad1 coexpression. Scale bars:100 $\mu \mathrm{m}$ (A-D) and $50 \mu \mathrm{m}$ (E and F).

cascades are also implicated in ALK signaling, in which TGF- $\beta$ activating kinase (TAK1), a member of the MAP kinase kinase kinase family, plays a key role. TAK1 activates MAP kinase kinase in combination with an adaptor molecule, TAB1, which leads to JNK and p38 activation (32). The role of p38 in chondrogenesis has recently attracted particular interest because p38 inhibitors such as SB203580 suppress the chondrogenic differentiation of ATDC5 cells induced by growth/differentiation factor-5 $(33,39)$. However, the exact roles of the Smad pathways and p38 pathways in chondrocyte differentiation are not yet fully clarified. We used a combination of adenoviral gene delivery and a chemical inhibitor to segregate the roles of these two pathways downstream of ALK3 activation and found that (a) inhibitory Smad (Smad6) expression or treatment with the p38 inhibitor SB203580 suppressed the effect of ALK3 ${ }^{\mathrm{CA}}$ expression (Figure 2) (b) Smad1 synergistically augmented the effect of ALK3 ${ }^{\mathrm{CA}}$ (Figure $5 \mathrm{~A}$ ), and (c) activation of p38 pathways alone by MKK6 ${ }^{\mathrm{CA}}$ expression induced the hypertrophic differentiation markers type $X$ collagen and mineralization in SFs, which was suppressed by Smad 1 coexpression (Figures 5B and 6). These results suggest that although both Smad and p38 activation is necessary for chondrogenic differentiation of SFs, sustained activation of p38 pathways alone prompts the terminal differentiation of the cells. Consistent with our results, Zhen et al. (40) reported that parathyroid hormone inhibits type $X$ collagen expression in hypertrophic chondrocytes by suppressing p38 pathways. Von der Mark et al. (41) reported the focal appearance of type X collagen in osteoarthritic cartilage, which may be involved in the degenerative changes of the articular cartilage and in the pathogenesis of osteoarthritis. Using the mouse model of osteoarthritis, we found that activated p38 is associated with type X colla- 

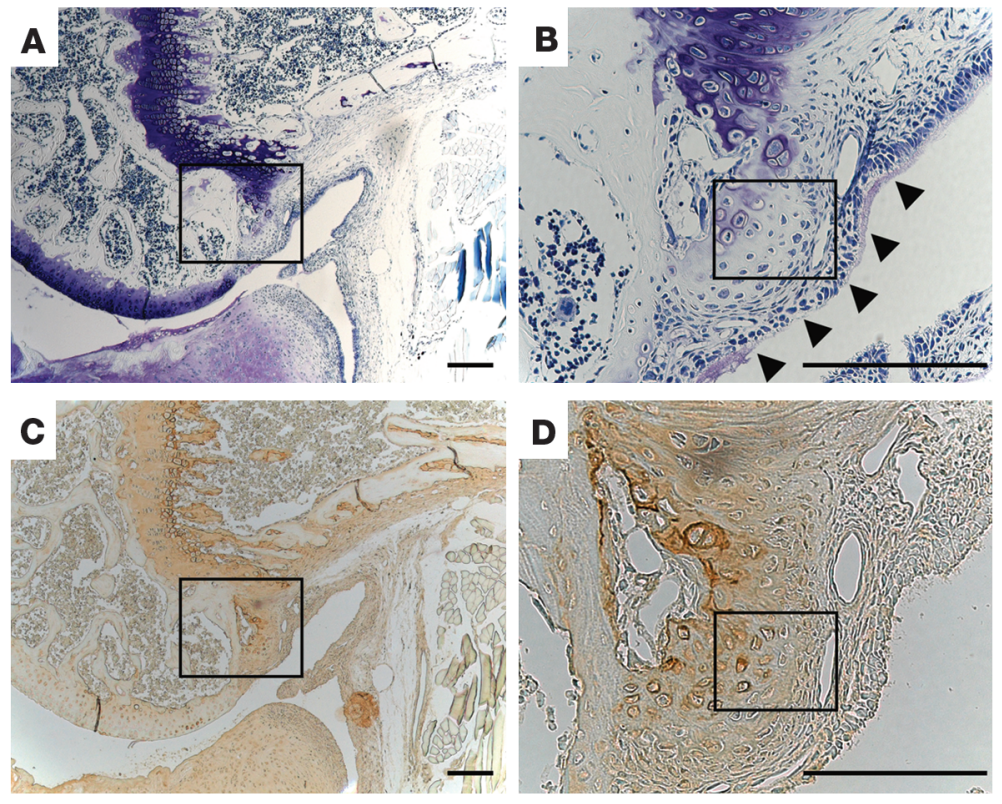

p-p38
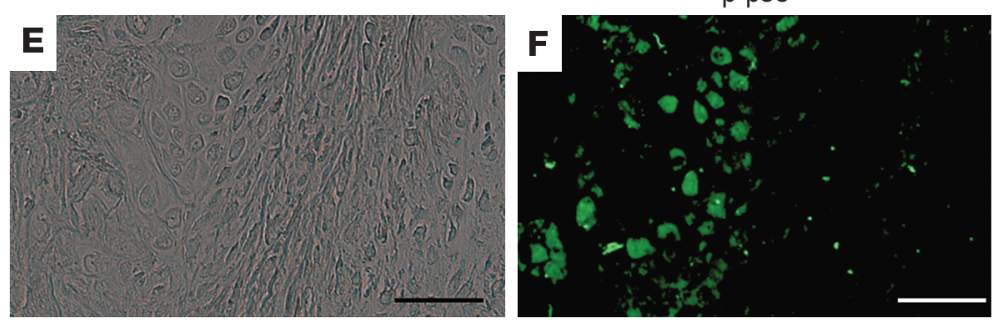

\section{Figure 7}

Histological analysis of knee joints in the mouse ACL and MM resection model. (A-F) Toluidine blue staining ( $\mathbf{A}$ and $\mathbf{B})$ and type $\mathbf{X}$ collagen immunostaining $(\mathbf{C}$ and $\mathbf{D})$ at the marginal area between the articular cartilage and synovium. B and D present higher-magnification views of $\mathbf{A}$ and $\mathbf{C}$, respectively. Osteochondrophytes were formed at the posterior edge of the femoral condyle, and they were positively stained by anti-type $X$ collagen as well as toluidine blue (rectangular areas in $\mathbf{A}$ and $\mathbf{C}$ ). Clusters of migrating synovial cells were observed adjacent to the osteochondrophytes (B, arrowheads) where future osteochondrophytes will develop, and they were positively stained by anti-type $\mathrm{X}$ collagen at the marginal area between synovium and osteophytes (rectangular area in $\mathbf{D}$ ). This region was also positively stained by anti-phospho-p38 (F). E and $\mathbf{F}$ represent phase-contrast microscopy (E) and immunostaining with anti-phospho-p38 (F) of the rectangular area in D. Positive phospho-p38 staining was observed at the area of osteochondrophytes as well as the marginal synovium. Scale bars: $500 \mu \mathrm{m}$ (A-D) and $50 \mu \mathrm{m}$ (E and F). gen expression in the synovial tissues adjacent to osteochondrophytes as well as in the degenerative cartilage (Figure 7).

Smad pathways not only are required for chondrogenic differentiation of SFs but also critically regulate the stage of differentiation of the cells and suppress their terminal differentiation process. Consistent with our findings, Scharstuhl recently reported inhibitory action of Smad7 in TGF- $\beta$-induced chondrocyte proliferation and proteoglycan production (42), indicating a critical role for Smad pathways. Hidaka and coworkers (43) demonstrated that adenovirus vector-mediated BMP-7 expression in chondrocytes accelerates the cartilage repair process. More recently, Lories and colleagues (44) demonstrated that BMP-2 and BMP-6 expressed in arthritic synovium are regulated by proinflammatory cytokines and differentially modulate fibroblast-like synoviocyte apoptosis, and Fukui et al. (45) found that BMP-2 expression was increased by proinflammatory cytokines in normal and osteoarthritis chondrocytes. These findings, combined with our observations, suggest that although BMPs have favorable effects on the repair process of articular cartilage, they may have proapoptotic and/or degenerative effects on the cells when $\mathrm{p} 38$ pathways are overactivated. Our findings suggest an important role for p38 signal transduction pathways in chondrocytes and SFs, leading to degenerative joint disorders, and suggest the potential utility of p38 modifiers in the treatment of rheumatoid arthritis and/or osteoarthritis. In fact, p38 kinase modifiers are now in clinical trials to treat rheumatoid arthritis (46). Based on our observations, we would like to propose that SFs are an excellent source for chondroprogenitors, which can be differentiated into chondrocytes via ALK3 activation, and that activation of the Smad pathway while controlling the degree of p38 activation may be a way to generate committed chondrocytes for the repair and/or replacement of cartilage.

\section{Acknowledgments}

The authors thank R. Yamaguchi and M. Ikeuchi (Department of Orthopaedic Surgery, The University of Tokyo), who provided expert technical assistance; K.L. Insogna (Yale University) for critical reading of the manuscript; and Y. Iwamoto (Thomas Jefferson University) for type II collagen and aggrecan probes. ALK and Smad adenovirus vectors were kindly provided by K. Miyazono (The University of Tokyo) and T. Imamura (The Cancer Institute of the Japanese Foundation for Cancer Research). This work was in part supported by Grants-in-Aid from the Ministry of Education, Culture, Sports, Science and Technology of Japan, Health Science research grants from the Ministry of Health and Welfare of Japan and an Uehara Memorial Award to S. Tanaka.

Received for publication August 28, 2003, and accepted in revised form January 6, 2004.

Address correspondence to: Sakae Tanaka, Department of Orthopaedic Surgery, Faculty of Medicine, The University of Tokyo, 7-3-1 Hongo, Bunkyo-ku, Tokyo 113-0033, Japan. Phone: 81-3-3815-5411 ext. 33376; Fax: 81-3-3818-4082; E-mail: TANAKAS-ORT@h.u-tokyo.ac.jp. 
1. Hunziker, E.B. 2002. Articular cartilage repair: basic science and clinical progress. A review of the current status and prospects. Osteoarthritis Cartilage. 10:432-463.

2. Poole, A.R., et al. 2001. Composition and structure of articular cartilage: a template for tissue repair. Clin. Orthop. 391:S26-S33.

3. Poole, A.R. 2003. What type of cartilage repair are we attempting to attain? J. Bone Joint Surg. Am. 85-A(Suppl. 2):40-44

4. Hunziker, E.B. 2003. Tissue engineering of bone and cartilage. From the preclinical model to the patient. Novartis Found. Symp. 249:70-85, 170-174, and 239-141.

5. Bruder, S.P., et al. 1998. Mesenchymal stem cells in osteobiology and applied bone regeneration. Clin. Orthop. 355:S247-S256.

6. Matsusue, Y., Yamamuro, T., and Hama, H. 1993. Arthroscopic multiple osteochondral transplantation to the chondral defect in the knee associated with anterior cruciate ligament disruption. Arthroscopy. 9:318-321.

7. Bentley, G., et al. 2003. A prospective, randomised comparison of autologous chondrocyte implantation versus mosaicplasty for osteochondral defects in the knee. J. Bone Joint Surg. Br. 85:223-230.

8. Peterson, L., Minas, T., Brittberg, M., and Lindahl, A. 2003. Treatment of osteochondritis dissecans of the knee with autologous chondrocyte transplantation: results at two to ten years. J. Bone. Joint Surg. Am. 85-A(Suppl 2):17-24.

9. Peterson, L., Brittberg, M., Kiviranta, I., Akerlund, E.L., and Lindahl, A. 2002. Autologous chondrocyte transplantation. Biomechanics and long-term durability. Am. J. Sports Med. 30:2-12.

10. Minas, T., and Nehrer, S. 1997. Current concepts in the treatment of articular cartilage defects. Orthopedics. 20:525-538.

11. Hangody, L., Feczko, P., Bartha, L., Bodo, G., and Kish, G. 2001. Mosaicplasty for the treatment of articular defects of the knee and ankle. Clin. Orthop. 391:S328-S336.

12. Firestein, G.S. 2003. Evolving concepts of rheumatoid arthritis. Nature. 423:356-361.

13. Takayanagi, H., et al. 1999. Suppression of arthritic bone destruction by adenovirus-mediated csk gene transfer to synoviocytes and osteoclasts. J. Clin. Invest. 104:137-146.

14. Hunziker, E.B., and Rosenberg, L.C. 1996. Repair of partial-thickness defects in articular cartilage: cell recruitment from the synovial membrane. J. Bone Joint Surg. Am. 78:721-733.

15. Nishimura, K., et al. 1999. Chondroprogenitor cells of synovial tissue. Arthritis Rheum. 42:2631-2637.

16. De Bari, C., Dell'Accio, F., Tylzanowski, P., and Luyten, F.P. 2001. Multipotent mesenchymal stem cells from adult human synovial membrane. Arthritis Rheum. 44:1928-1942.
17. De Bari, C., et al. 2003. Skeletal muscle repair by adult human mesenchymal stem cells from synovial membrane. J. Cell Biol. 160:909-918.

18. O'Connell, J.X. 2000. Pathology of the synovium. Am. J. Clin. Pathol. 114:773-784.

19. Wozney, J.M. 1989. Bone morphogenetic proteins. Prog. Growth Factor Res. 1:267-280.

20. Kulyk, W.M., Rodgers, B.J., Greer, K., and Kosher, R.A. 1989. Promotion of embryonic chick limb cartilage differentiation by transforming growth factor-beta. Dev. Biol. 135:424-430.

21. Lafeber, F.P., Vander Kraan, P.M., Van Roy, J.L., Huber-Bruning, O., and Bijlsma, J.W. 1993. Articular cartilage explant culture; an appropriate in vitro system to compare osteoarthritic and normal human cartilage. Connect. Tissue Res. 29:287-299.

22. Denker, A.E., Nicoll, S.B., and Tuan, R.S. 1995. Formation of cartilage-like spheroids by micromass cultures of murine C3H10T1/2 cells upon treatment with transforming growth factor- $\beta 1$. Differentiation. 59:25-34.

23. Yamamoto, A., et al. 2003. Suppression of arthritic bone destruction by adenovirus-mediated dominant-negative Ras gene transfer to synoviocytes and osteoclasts. Arthritis Rheum. 48:2682-2692.

24. Takayanagi, H., et al. 2000. Involvement of receptor activator of nuclear factor $\kappa \mathrm{B}$ ligand/osteoclast differentiation factor in osteoclastogenesis from synoviocytes in rheumatoid arthritis. Arthritis Rheum. 43:259-269.

25. Tanaka, S., et al. 1998. Modulation of osteoclast function by adenovirus vector-induced epidermal growth factor receptor. J. Bone Miner. Res. 13:1714-1720.

26. Miyake, S., et al. 1996. Efficient generation of recombinant adenoviruses using adenovirus DNAterminal protein complex and a cosmid bearing the full-length virus genome. Proc. Natl. Acad. Sci. U. S. A. 93:1320-1324.

27. Fujii, M., et al. 1999. Roles of bone morphogenetic protein type I receptors and Smad proteins in osteoblast and chondroblast differentiation. Mol. Biol. Cell. 10:3801-3813.

28. Enomoto-Iwamoto, M., et al. 2000. Hedgehog proteins stimulate chondrogenic cell differentiation and cartilage formation. J. Bone Miner. Res. 15:1659-1668.

29. Kamekura, S., et al. 2003. Establishment of novel experimental osteoarthritis models in mice. J. Bone Miner. Res. 18:S395.

30. van den Berg, W.B. 2001. Lessons from animal models of osteoarthritis. Curr. Opin. Rheumatol. 13:452-456

31. Derynck, R., Zhang, Y., and Feng, X.H. 1998. Smads: transcriptional activators of TGF- $\beta$ responses. Cell. 95:737-740.

32. Miyazono, K., Kusanagi, K., and Inoue, H. 2001. Divergence and convergence of TGF- $\beta / B M P$ signaling. J. Cell Physiol. 187:265-276.
33. Watanabe, H., de Caestecker, M.P., and Yamada, Y. 2001. Transcriptional cross-talk between Smad, ERK1/2, and p38 mitogen-activated protein kinase pathways regulates transforming growth factor- $\beta$ induced aggrecan gene expression in chondrogenic ATDC5 cells. J. Biol. Chem. 276:14466-14473.

34. Miyazono, K. 1997. TGF- $\beta$ receptors and signal transduction. Int. J. Hematol. 65:97-104.

35. Massague, J. 1998. TGF- $\beta$ signal transduction. Annu. Rev. Biochem. 67:753-791.

36. de Crombrugghe, B., et al. 2000. Transcriptional mechanisms of chondrocyte differentiation. Matrix Biol. 19:389-394.

37. Akiyama, H., Chaboissier, M.C., Martin, J.F., Schedl, A., and de Crombrugghe, B. 2002. The transcription factor Sox 9 has essential roles in successive steps of the chondrocyte differentiation pathway and is required for expression of Sox 5 and Sox6. Genes Dev. 16:2813-2828.

38. Mi, Z., et al. 2003. Adverse effects of adenovirusmediated gene transfer of human transforming growth factor beta 1 into rabbit knees. Arthritis Res. 5:R132-R139.

39. Nakamura, K., et al. 1999. p38 mitogen-activated protein kinase functionally contributes to chondrogenesis induced by growth/differentiation factor-5 in ATDC5 cells. Exp. Cell Res. 250:351-363.

40. Zhen, X., Wei, L., Wu, Q., Zhang, Y., and Chen, Q. 2001. Mitogen-activated protein kinase p38 mediates regulation of chondrocyte differentiation by parathyroid hormone. J. Biol. Chem. 276:4879-4885

41. von der Mark, K., et al. 1995. Upregulation of type $\mathrm{X}$ collagen expression in osteoarthritic cartilage. Acta Orthop. Scand. Suppl. 266:125-129.

42. Scharstuhl, A., et al. 2003. Adenoviral overexpression of Smad-7 and Smad- 6 differentially regulates TGF- $\beta$-mediated chondrocyte proliferation and proteoglycan synthesis. Osteoarthritis Cartilage. 11:773-782.

43. Hidaka, C., et al. 2003. Acceleration of cartilage repair by genetically modified chondrocytes over expressing bone morphogenetic protein-7. J. Orthop. Res. 21:573-583.

44. Lories, R.J., Derese, I., Ceuppens, J.L., and Luyten, F.P. 2003. Bone morphogenetic proteins 2 and 6 , expressed in arthritic synovium, are regulated by proinflammatory cytokines and differentially modulate fibroblast-like synoviocyte apoptosis. Arthritis Rheum. 48:2807-2818.

45. Fukui, N., Zhu, Y., Maloney, W.J., Clohisy, J., and Sandell, L.J. 2003. Stimulation of BMP-2 expression by pro-inflammatory cytokines IL-1 and TNF- $\alpha$ in normal and osteoarthritic chondrocytes. J. Bone Joint Surg. Am. 85-A(Suppl. 3):59-66.

46. Pargellis, C., and Regan, J. 2003. Inhibitors of p38 mitogen-activated protein kinase for the treatment of rheumatoid arthritis. Curr. Opin. Investig. Drugs. 4:566-571. 